\title{
Por un feminismo radical y plural: Repensando las coordenadas teóricas y políticas de un nuevo feminismo desde una lectura cruzada de Judith Butler, Ernesto Laclau y Chantal Mouffe*
}

Malena Nijensohn**

\begin{abstract}
Resumen
En el presente artículo me propongo analizar los límites de una concepción liberal del feminismo y los movimientos LGBTIQ+, basada en la identidad y la autonomía, que centra la lucha política en la demanda de derechos. A través del análisis del feminismo de los últimos años en Argentina (a partir del fenómeno $\mathrm{Ni}$ Una Menos), propongo un feminismo al que llamo "radical y plural": un feminismo que tome la vulnerabilidad como punto de partida de la reflexión política y que, a través de la movilización de dicha vulnerabilidad, se proponga profundizar los valores democráticos de libertad $e$ igualdad.
\end{abstract}

Palabras clave: Feminismo, Democracia Radical y Plural, Performatividad Plural, Movilizaciones, Ni Una Menos.

* Recibido el 11 de diciembre de 2017, aceptado el 19 de septiembre de 2018. ** Doctoranda en Estudios de Género, Facultad de Filosofía y Letras, Universidad de Buenos Aires, Buenos Aires, Argentina. malenanijensohn@gmail.com 
For a Radical and Plural Feminism: Thinking Theoretical and Politic Coordinates of a New Feminism from a Cross-Reading of Judith Butler, Ernesto Laclau and Chantal Mouffe

\begin{abstract}
In this article, I propound an analysis of the limits of a liberal conception of feminism and LGBTIQ+ movements, based on identity and autonomy, and whose struggle is centered in the claim of rights. Through the analyses of the feminist movement of the last years in Argentina (since the "Ni Una Menos" phenomenon), I propound a feminism that I call "radical and plural": a feminism that takes vulnerability as the point of departure for political thinking, and that, through the mobilisation of that vulnerability, aims for a deepening of the democratic values of liberty and equality.
\end{abstract}

Keywords: Feminism, Radical and Plural Democracy, Plural Performativity, Mobilizations, Ni Una Menos. 


\section{Introducción}

En el prólogo a la traducción al castellano de su último libro, Fortunas del feminismo, Nancy Fraser sostiene que el feminismo de la segunda ola que, inicialmente, había intentado transformar la sociedad desde la raíz, fue poco a poco encausando sus luchas políticas bajo la modalidad de las políticas identitarias, pasando de la redistribución al reconocimiento y, por tanto, centrándose en la política cultural más que en la económica. ${ }^{1} \mathrm{~A}$ sus ojos, en el marco del neoliberalismo de los años ochenta y su proyecto de libre mercado contrario a la idea de la redistribución igualitaria, el feminismo que antes se había apoyado en el Estado de bienestar para ampliar los valores igualitarios de la clase al género opera un giro que lo lleva a una agenda centrada en la política del reconocimiento bajo la forma de la política de la identidad.

En el contexto de fin de siglo, el giro hacia el reconocimiento encajó con demasiada facilidad en un ascendente neoliberalismo que solo quería reprimir cualquier recuerdo del igualitarismo social. El resultado fue una trágica ironía histórica. En lugar de llegar a un paradigma más amplio y más rico, capaz de abarcar la redistribución y el reconocimiento, las feministas intercambiaron de hecho un paradigma truncado por otro: un economicismo truncado por un culturalismo truncado. (Fraser, 2015: 21)

A principios de 2017, en un artículo titulado "El final del neoliberalismo "progresista", Fraser va aún más lejos y sostiene

\footnotetext{
1 Nancy Fraser diferencia entre dos formas de lucha: luchas por la redistribución (que remiten a injusticias socioeconómicas) y luchas por el reconocimiento (que remiten a injusticias de tipo social o cultural). La autora no cree que sean dos cuestiones que queden aisladas, pero las separa por cuestiones heurísticas. Al respecto, ver: Fraser (1997). Para un debate acerca de la diferenciación entre las injusticias socio económicas y las injusticias culturales, ver Butler (1997).
} 
que el régimen actual no es el neoliberalismo sin más, sino el neoliberalismo progresista:
una alianza de las corrientes principales de los nuevos movimientos sociales (feminismo, antirracismo, multiculturalismo y derechos de los LGBTQ), por un lado, y, por el otro, sectores de negocios de gama alta "simbólica" y sectores de servicios (Fraser, 2017).

Siguiendo su diagnóstico, los nuevos movimientos sociales les habrían dado un aire progresista a las medidas neoliberales financieras de precarización y austeridad. Un ejemplo podría ser el pequeño sector de mujeres privilegiadas que ascendió en puestos empresariales, como si allí se jugara el empoderamiento y la autonomía feministas. Sobre estos conceptos (empoderamiento y autonomía) volveré más adelante.

Considero, junto a Fraser, que es menester repensar la forma que tomó el feminismo en términos de lucha por el reconocimiento bajo la modalidad de la política de la identidad, aunque no comparto con ella que redistribución y reconocimiento sean dos cuestiones separadas $y$, por tanto, que el feminismo se haya vuelto "meramente cultural", por retomar la expresión de Judith Butler en debate con Fraser (Butler, 1997), así como tampoco creo que se pueda subsumir todo el feminismo actual bajo el feminismo liberal. Finalmente, considero menester repensar qué tipo de feminismo es necesario hoy en día y desde qué coordenadas teóricas y militantes lo sostenemos. Como se verá a lo largo del presente trabajo, propongo un retorno a la cuestión de la igualdad y la justicia social, pero lo hago desde otras coordenadas teóricas, más fructíferas a mi entender para comprender la lógica política contemporánea y el feminismo que se está gestando en Argentina. ${ }^{2}$

\footnotetext{
2 Para una lectura crítica de la hipótesis de Nancy Fraser respecto de la afinidad entre el feminismo y el neoliberalismo y su aplicabilidad a las coordenadas latinoamericanas, ver: Schild (2016).
} 
El trabajo está dividido en dos partes. En primer lugar, (I) voy a desarrollar una crítica al feminismo mainstream (es decir al feminismo blanco, heterosexual, de clase media) a través de (a) la búsqueda de un feminismo no esencialista, que parta de la vulnerabilidad constitutiva de la subjetividad y (b) de la reflexión sobre la concepción desarrollada por Leticia Sabsay de que el sujeto sexual se ha constituido como "sujetos de derechos", para sopesar las potencias y los límites de esta forma de lucha política. En segundo lugar, (II) voy a esbozar la idea de un feminismo radical y plural, basándome en (a) las ideas de Ernesto Laclau y Chantal Mouffe sobre hegemonía y democracia radical y plural y (b) el desarrollo de Judith Butler acerca de la performatividad plural de los cuerpos aliados en las manifestaciones en el espacio público. Esto me permitirá efectuar una crítica al feminismo neoliberal, con la esperanza de que un feminismo de articulaciones radicales y plurales pueda adquirir centralidad como forma de resistencia a las tecnologías neoliberales de gobierno.

\section{Crítica al feminismo mainstream}

a. Emergencia del "proletariado del feminismo". De la identidad a la vulnerabilidad.

Dice Paul Preciado en Testo Yonki respecto del feminismo crítico que pone en cuestión que el sujeto del feminismo sean las mujeres, en tanto que cis-mujeres, heterosexuales, blancas y de clase media:

Estos feminismos disidentes se hacen visibles a partir de los años ochenta, cuando, en sucesivas oleadas críticas, los sujetos excluidos por el feminismo biempensante comienzan a criticar los procesos de purificación y la represión de sus proyectos revolucionarios que ha conducido hasta un feminismo gris, normativo y puritano que ve en las diferencias culturales, sexuales o políticas amenazas a su ideal heterosexual y eurocéntrico de mujer. Se trata de lo que podríamos llamar, tomando la expresión de Virginie 
Despentes, el despertar crítico del 'proletariado del feminismo', cuyos malos sujetos son las putas, las lesbianas, las violadas, los cis-hombres disidentes, las marimachos, los y las transexuales, las mujeres que no son blancas, las musulmanas..., en definitiva, casi todos nosotros (Preciado, 2014:263)

Si bien Butler no es la primera en darse cuenta de esto, sino que la estadounidense se pliega más bien a una serie de críticas que los movimientos feministas disidentes (chicano, gay, lésbico, queer, negro, etc.) venían señalando desde los años setenta y ochenta, sí podemos afirmar que, de alguna forma, su libro Gender Trouble sintetiza estas diferentes críticas efectuando un giro radical al interior del movimiento (tanto en el ala teórica como en la militante). En primer lugar, señala Butler, la categoría mujeres es "un término problemático, un sitio de disputa, una causa de ansiedad" (2007:4, la traducción es mía). ${ }^{3}$ Esto, de alguna forma u otra, es lo que el feminismo de la segunda ola ya había expuesto, en la medida en que cuestionaba el rol social asignado a las mujeres. ${ }^{4}$ La presuposición de universalidad y estabilidad del sujeto "mujeres" responde a ciertas restricciones dependientes de la política clásica de la representación, según la cual es menester que haya sujetos preestablecidos cuyos intereses son representados. Así, y esta es la segunda cuestión y lo que marcará el pasaje de la segunda a la tercera ola del feminismo, la insistencia en un sujeto estable "mujeres" sin fisuras genera múltiples rechazos $y$ exclusiones, tornándose coactiva $y$ reguladora. ¿Qué presupuestos de raza, clase, biológicos, económicos, culturales están en juego en la constitución de las

\footnotetext{
3 "a troublesome term, a site of contest, a cause for anxiety".

4 A partir de la consigna beauvoiriana "no se nace mujer: se llega a serlo" (De Beauvoir, 2012:207) se conforma una concepción del género cultural como algo diferenciado del sexo biológico. Aunque la francesa no hable de "género", es posible leer aquí la idea de que no hay una única forma de llegar a ser mujer, en el sentido de que no hay un determinismo signado biológicamente en el nacimiento.
} 
"mujeres" como sujeto del feminismo? ¿Sobre qué exclusiones se funda el concepto "mujeres"? Dice Butler: "Quizás el problema sea incluso más serio." Tercera cuestión.

\begin{abstract}
¿La construcción de la categoría de mujer como un sujeto coherente y estable es una regulación y una reificación involuntarias de las relaciones de género? ¿Y tal reificación no está precisamente en contra de los objetivos feministas? ¿Hasta qué punto la categoría de mujer obtiene estabilidad y coherencia sólo en el contexto de la matriz heterosexual? (Butler, 2007:7, la traducción es mía). ${ }^{5}$
\end{abstract}

El término "matriz heterosexual" designa la grilla de inteligibilidad a través de la cual se naturalizan los cuerpos, los géneros y los deseos y prácticas sexuales. La matriz heterosexual produce a los sujetos bajo la pretensión de un vínculo causal que va desde el sexo (en tanto que dato biológico, bajo la división binaria macho/hembra) hacia el género (como producción cultural de feminidad y masculinidad) y finalmente hacia el deseo y las prácticas (hetero)sexuales. Así, la heterosexualidad en tanto que institución requiere y produce a un tiempo la univocidad de cada uno de estos términos dentro de un sistema oposicional binario: macho/hembra, varón/mujer y deseo y práctica heterosexual bajo la ley de la complementariedad de los sexos. Entonces, si "mujeres" sólo adquiere sentido dentro de la matriz heterosexual, ¿no reproduce el feminismo, de alguna forma, dicha matriz al invocar al sujeto mujeres sin más?

Lo que Butler se propone desmontar es la distinción propia del feminismo de la segunda ola entre sexo y género, ya que para la estadounidense la idea del sexo como algo prediscursivo es una producción discursiva. Así, no hay un sexo como hecho natural,

5 "Perhaps the problem is even more serious. Is the construction of the category of women as a coherent and stable subject an unwitting regulation and reification of gender relations? And is not such a reification precisely contrary to feminists aims? To what extent does the category of women achieve stability and coherence only in the context of the heterosexual matrix?". 
sino que éste está siempre mediado por una interpretación generizada. No hay una identidad previa a los mecanismos discursivos de representación, sino que las relaciones de poder producen a los sujetos que más tarde dicen representar. Así, para Butler la identidad se produce performativamente, en el sentido de que "no hay identidad de género detrás de las expresiones de género; esa identidad es constituida performativamente por las mismas 'expresiones' que dicen ser el resultado de ésta" (Butler, 2007:34, la traducción es mía). ${ }^{6}$ Entender la identidad como una construcción performativa significa que ésta se instituye en la repetición estilizada de palabras, actos, gestos y estilos performativos y es el efecto creado por tales performativos.

Para Butler, la identidad no puede ser la premisa de las coaliciones políticas y el "nuevo feminismo", que interroga las prácticas reguladoras de la formación del género y analiza cómo éstas constituyen las identidades y la supuesta coherencia interna de los sujetos, será un feminismo que no se fundará en el sujeto "mujeres". Entonces, ¿a partir de dónde pensar las nuevas coaliciones políticas? En su último libro, Notes toward a performative theory of assembly, Butler dice:

Ahora estoy trabajando la cuestión de las alianzas entre
varias minorías o poblaciones consideradas desechables;
más específicamente, me interesa cómo la precaridad -ese
término medio, en algunas maneras, ese término que
media- puede operar, y opera, como sitio de alianza entre
grupos de gente que de otra forma no tendría mucho en

6 "There is no gender identity behind the expressions of gender; that identity is performatively constituted by the very 'expressions' that are said to be its results".

7 En una entrevista realizada por Patricia Soley-Beltran y Paul B. Preciado en 2006, Judith Butler explicaba en qué sentido la pregunta por el "sujeto del feminismo" restringía la potencia política del movimiento. Cfr. Soley-Beltran, Preciado (2006:220): "No se necesita saber cuál es el sujeto del feminismo para estar en esa lucha, y creo que en el momento en que nos atascamos en la cuestión de quién es el sujeto del feminismo, nos olvidamos de cuáles son las cuestiones políticas más amplias". 
común $y$ entre quienes hay incluso desconfianza $y$ antagonismo (Butler, 2015:27, la traducción es mía). ${ }^{8}$

Así, tomando la vulnerabilidad como punto de partida de la reflexión política, sostiene Butler, se puede correrse del eje de las políticas de la identidad. Y la vulnerabilidad aparece como el punto de partida de la vida política en la medida en que consideremos nuestra dependencia fundamental de otros, en el sentido de que la vida corporal está siempre expuesta y abierta a los otros. Butler nombra a esta condición fundamental precariedad (precariousness); ahora bien, que toda vida sea precaria no significa que todas las vidas sean precarias en el mismo sentido: la precaridad (precarity) designa la distribución diferencial de la precariedad, una condición inducida políticamente, que produce algunas vidas más vulnerables que otras, en la medida en que no acceden a las redes económicas y sociales de contención.

De alguna forma, todos vivimos con esta particular vulnerabilidad, una vulnerabilidad hacia lo otro que es parte de la vida corporal, una vulnerabilidad a una llamada repentina de otra parte que no podemos anticipar. Esta vulnerabilidad, sin embargo, se vuelve altamente exacerbada bajo ciertas condiciones sociales y políticas, especialmente aquellas en las que la violencia es una forma de vida y los medios para asegurar la auto-defensa están limitados (Butler, 2006:29, la traducción es mía). ${ }^{9}$

\footnotetext{
8 "Now I am working the question of alliances among various minorities or populations deemed disposable; more specifically, I am concerned with how precarity -that middle term, in some ways, that mediating term-might operate, or is operating, as a site of alliance among groups of people who do not otherwise find much in common and between whom there is sometimes even suspicion and antagonism".

9 "In a way, we all live with this particular vulnerability, a vulnerability to the other that is part of bodily life, a vulnerability to a sudden address from elsewhere that we cannot preempt. This vulnerability, however, becomes highly exacerbated under certain social and political conditions, especially those in which violence is a way of life and the means to secure self-defense are limited".
} 
Ahora bien, tomar la vulnerabilidad como punto de partida para pensar la resistencia puede sonar paradójico, pero esto sólo tiene lugar si se aceptan dos presupuestos básicos que aquí serán rechazados y que se asientan en la asociación de la vulnerabilidad con la inacción. Estos dos presupuestos son la idea de que la vulnerabilidad (como lugar de victimización y pasividad) es lo opuesto de la resistencia y la idea de que, por lo tanto, necesita formas de protección paternalistas (que son el lugar de la agencia) (Butler, Sabsay, Gambetti, 2016:1). Laura Lowenkron, en un artículo donde analiza el pasaje del uso de la categoría de consentimiento hacia el de la categoría de vulnerabilidad en el vocabulario jurídico en lo concerniente a la violencia sexual, sostiene que con la introducción de la variable de la vulnerabilidad se reduce la agencia de las personas vulnerables y se las condena a la pasividad (Lowenkron, 2015). ${ }^{10}$ Esta asociación de la vulnerabilidad con la pasividad y la incapacidad de acción es lo que Butler se propone deconstruir, para pensar una noción de agencia que no se desentienda de la interdependencia, sin por ello dar lugar a una demanda de políticas públicas o leyes jurídicas paternalistas. Pues como sostienen Butler, Gambetti y Sabsay, muchas veces el feminismo otorga a instituciones sociales o políticas paternalistas la capacidad de llevar a cabo objetivos feministas, o incluso lleva a cabo prácticas que reponen las lógicas paternalistas, considerando

\footnotetext{
${ }^{10} \mathrm{El}$ argumento de Lowenkron se centra en la legislación de dos formas de violencia sexual: por un lado, el abuso sexual infantil y, por el otro, la trata de personas con fines de explotación sexual. En el caso de las relaciones sexuales con menores de edad, se trata siempre de una violación del vulnerable, independientemente de la presencia o ausencia de consentimiento. Se asocia la vulnerabilidad a la minoría de edad, a la enfermedad mental y a la incapacidad de ofrecer resistencia. En el caso de la trata de personas, puesto que la ley brasileña no toma en cuenta el consentimiento, Lowenkron analiza el Protocolo de Palermo, donde la coerción es pensada como el abuso de autoridad o de una posición de vulnerabilidad. Así, la vulnerabilidad aparece como el locus de la pasividad y de la victimización. Esta (re)esencialización del término "vulnerabilidad" es algo que, a los ojos de la brasileña, también hace Butler, en la medida en que considera la vulnerabilidad (la "precariousness") como una condición humana compartida por todos los seres en tanto que seres sociales.
} 
a algunas mujeres como víctimas desde la perspectiva de una vulnerabilidad que las deja incapacitadas para la agencia. Aquí, por el contrario, intentaré pensar la vulnerabilidad como la condición de posibilidad de la resistencia y buscaré modos feministas de agencia que no repongan la concepción moderna masculinista de la acción política.

Todo esto en un contexto de condiciones neoliberales de precarización y pauperización, que deja a ciertos sectores más expuestos a la pobreza, la violencia y la exclusión social. Si pensamos la precarización como una herramienta biopolítica de gobernanza neoliberal, a la luz de los últimos años se puede ver una intensificación de la precarización, así como procesos de pauperización acompañados de una criminalización de la protesta y la subsecuente intensificación de políticas securitarias (Sabsay, 2016:283-284). Esto nos lleva a la necesidad de buscar nuevas formas de resistencia que desafíen los límites de la democracia representativa. Una de ellas es la reflexión butleriana acerca de las asambleas públicas o la alianza de los cuerpos en el espacio público, cuestión que retomaré en la segunda sección de este artículo. En el próximo apartado me voy a centrar en algunos problemas políticos de las políticas de la identidad ya mencionadas, siguiendo las reflexiones de Leticia Sabsay en su último libro, The political imaginary of sexual freedom, porque, tal como ella dice: "El trabajo crítico que pretendía deconstruir [la figura del sujeto] fue hecho y podemos felicitarnos dentro de los muros de nuestra burbuja académica, pero el mundo continúa como siempre" (Sabsay, 2016b: 5, la traducción es mía). ${ }^{11}$

11 "The critical work that aimed to deconstruct it [the figure of the subject] has been done and we can congratulate ourselves within the walls of our academic bubble, but the world keeps going on as usual'. 
b. ¿Caben nuestros sueños en sus urnas? De la lengua jurídica a la potencia de la imaginación

Siguiendo a Leticia Sabsay, a principios de los años noventa se produjo un "nuevo giro sexual democrático", a saber: procesos tanto políticos como culturales a través de los cuales la idea de la libertad sexual asumió una nueva legitimidad basada en los derechos. Así, lo que en su momento se concibió como "liberación sexual", es decir, un desprendimiento y un cuestionamiento de las normas que gobernaban la sexualidad, comenzó a cruzarse con el discurso de los derechos, reinscribiéndose en la lógica jurídica. De alguna forma, asistimos desde los años ochenta a una articulación de las luchas feministas, gays, lésbicas, trans, LGBTI+ en términos de la demanda de derechos para los diversos colectivos bajo el paradigma de la democracia liberal. ¿Cómo debe concebirse el sujeto sexual para convertirse en un sujeto portador de derechos? ¿Cómo debe imaginarse la sexualidad para que se convierta en un derecho? ¿Qué se gana y qué se pierde en la inscripción de los ideales libertarios en el lenguaje jurídico?

Si se piensa en las luchas por el reconocimiento de los derechos LGBTI+ como derechos humanos, en términos de una demanda de legitimidad del derecho a disentir de las normas sexuales hegemónicas, se puede ver que esta articulación de las luchas se llevó a cabo en términos de la política de la identidad, la cual descansa en una ontología liberal. Esto no significa que se conciba la identidad como estable, sustancial, universal; por el contrario, el sujeto que demanda los derechos es un sujeto postesencialista, sin que esto signifique que las ideas de la identidad sobre las que descansa hayan abandonado el paradigma liberal del sujeto.

Las tendencias actuales mainstream hacia la democratización de la sexualidad están enmarcadas en un imaginario psicosocial configurado alrededor de la figura predominante del sujeto neoliberal post-esencialista que, sin embargo, es caracterizado como transparente y autónomo 
en el entendimiento de sí mismo (Sabsay, 2016b:5, la traducción es mía). ${ }^{12}$

Así, ciertas categorías liberales, actualizadas bajo la modalidad neo-liberal, persistieron y son las que dieron forma a las luchas políticas feministas, gays, lésbicas, LGBTI+. De alguna forma, estas luchas y demandas se han anclado sobre el imaginario psicosocial individualista neoliberal que nos gobierna

Una aclaración se impone para evitar malos entendidos: considero que estas luchas en términos de la demanda de derechos han sido $y$ son fundamentales $y$ muy beneficiosas en términos de reconocimiento político y de articulación de políticas públicas, especialmente en Argentina, en América Latina y el Caribe. Conquistas tales como matrimonio igualitario, ley de identidad de género y demandas como aborto legal, seguro y gratuito o cupo laboral trans son la prueba de que necesitamos hablar la lengua jurídica y de que en el marco de las instituciones estatales se juega la posibilidad de un acceso más igualitario a la salud, la educación, el trabajo, la vivienda. De hecho, como desarrollaré en la próxima sección, el feminismo que quiero proponer, heredero de la noción de democracia radical y plural de Laclau y Mouffe, no se propone romper con las instituciones democráticas sino profundizarlas y extenderlas a más y nuevas relaciones sociales. En ese sentido, no desestimo la lucha por la ampliación de derechos, sino que el punto que quiero destacar aquí es, tal como lo explicita Sabsay, el siguiente:

En la superficie pareciera haber una tendencia liberalizadora en términos de la expansión de libertades clave y la "aceptación" (o tolerancia) de la diversidad sexual y genérica [...]. Sin embargo, lo que estamos viendo es que los discursos, las leyes a las políticas ostensiblemente

12 "IClurrent mainstream trends toward the democratization of sexuality are framed by a psychosocial imaginary configured around the prevailing figure of a neoliberal post-essentialist subject who nevertheless is characterized as transparent and autonomous in its self-understanding". 
progresistas asociados a esta liberalización en realidad están instituyendo nuevos modos históricos de regulación sexual, que de hecho condicionan las maneras en que la sexualidad y el género se comprenden, perciben y experimentan actualmente (Sabsay, 2016b:8-9, la traducción es mía ${ }^{13}$

Si Butler explicaba en 1999, en el segundo Prefacio a Gender Trouble, que su objetivo no había sido prescribir una nueva forma de vida en términos de sexo-género sino, más bien, "abrir el campo de posibilidades para el género sin dictar qué tipo de posibilidades deben ser realizadas" (Butler, 2007:viii, la traducción es mía) ${ }^{14}$, ¿qué lugar hay para pensar en las nuevas formas de regulación que se impusieron a través de la liberalización de la sexualidad y el género? ¿La forma de abrir nuevas posibilidades para el género, anclada en un paradigma y una ontología liberales, no terminaron dictando el tipo de posibilidades que podían ser realizadas?

La crítica fundamental que ofrece Sabsay y que aquí quiero considerar es que la supuesta liberalización de la vida sexogenérica, al quedar reducida a cuestiones como autonomía y derechos humanos (por anclarse en una ontología liberal), en realidad se convierte en una nueva forma de regular la sexualidad al comprender la disidencia como un compendio de identidades cerradas. Así, a pesar del trabajo de deconstrucción de la matriz heterosexual, del esfuerzo por desnaturalizar y mostrar el carácter contingente, no necesario, de las identidades y de la lucha por

13 " $[\mathrm{O}] \mathrm{n}$ the surface there seems to be a liberalizing trend in terms of the expansion of key liberties and the 'acceptance' (or toleration) of sexual and tender diversity [...]. However, what we are seeing is that ostensibly progressive discourses, laws, and policies associated with this liberalization are actually instituting historically new modes of sexual regulation, which in fact condition the ways in which sexuality and gender are currently understood, perceived, and experienced".

14 "the point was not to prescribe a new gendered way of life that might then serve as a model for readers of the text. Rather, the aim of the text was to open up the field of possibilities for gender without dictating which kinds of possibilities ought to be realized". 
abrir el campo de posibilidades vitales y afectivas, pareciera ser que la condición de ser sujeto y la identidad quedan ligadas a posiciones fijas, reconocibles, estables, inequívocas. Ahora el mercado, la industria cultural y hasta el activismo político nos ofrecen una serie de identidades para habitar. Nos encontramos en realidad con una diversificación de las posiciones posibles, que no por ser más dejan de estar gobernadas por las relaciones de poder. De esta forma, la heteronorma sigue ocupando un lugar central en los marcos progresistas, en la medida en que lo que han hecho es incluir ciertas identidades que antes eran excluidas y normalizar ciertas posiciones queer como conjunto de identidades estables, unívocas, discernibles.

Insisto, no se trata aquí de ofrecer una crítica sin más a las demandas por derechos fundadas en una concepción liberal del sujeto, sino de comprender cuáles son los riesgos y las limitaciones de las mismas a la hora de subvertir el heterocentrismo, si no se cuestiona ni el marco que posibilita la aparición de las diferencias ni la forma que ciertas diferencias deben asumir para poder ser incluidas dentro del mapa político de las identidades representables. ¿Es posible pensar en otro tipo de articulación política que no se limite a ampliar el mapa de posiciones posibles, sino que se aboque a efectuar una crítica de los valores (neo)liberales que moldean la identidad?

Los desplazamientos operados en el marco de la diversidad se reiteran sin desafiar la investidura de la concepción individualista y volitiva de la autonomía: el individuo liberal auto-suficiente como sujeto político (de voluntad $\mathrm{y}$ conciencia) se constituye a través del establecimiento de límites diferenciadores que estabilizan su posicionamiento, que a su vez mantiene la idea de libertad y apoya la demanda de derechos. Este mapa político centrado en la identidad se relaciona directamente con la concepción de la autonomía como materia de control sobre nuestros cuerpos. En este sentido, los límites que configuran el individuo autónomo establecen el estatus ontológico del cuerpo como un objeto (o entidad) cerrado, claro y distinto o incluso, 
como en los marcos neoliberales, como una empresa (Sabsay, 2016b:67-68, la traducción es mía ${ }^{15}$

Si la tarea deconstructiva se encargó de señalar el carácter construido de la subjetividad, la reapropiación neoliberal del constructivismo convirtió al sujeto en una empresa personal, es decir, en aquel que, a partir de una supuesta transparencia absoluta logra interpretarse a sí mismo y producir su propia verdad como el propietario absoluto de sus deseos. Aquí los ejemplos son múltiples, pero querría centrarme en la figura de la mujer empoderada, a saber: aquella mujer que a partir de una vivencia o una serie de vivencias logra transformar su situación de vulnerabilidad y se convierte así en protagonista de su propia historia. Aquí reaparecen todos los elementos propios de la concepción neoliberal: la asociación de la vulnerabilidad con la del rol de víctima pasiva y la agencia como la capacidad que se adquiere al superar dicha vulnerabilidad y adquirir el poder de producirse a uno mismo, donde la construcción del "yo" se basa en la concepción empresarial del sujeto. La comprensión de la libertad en términos de autonomía nos devuelve una subjetividad que es transparente $y$ propietaria de sí, que puede elegir libremente $y$ tiene la capacidad de hacerse $y$ deshacerse voluntariamente. Esta concepción nos aleja de aquello que Butler llamó "deshacer el género", es decir, de la idea de que "[n]i mi sexualidad ni mi género son precisamente una posesión, sino que ambos deben ser entendidos como maneras de ser desposeído, maneras de ser para otro o, de hecho, en virtud de otro" (Butler,

15 "The displacements that the framework of diversity operates reiterate without challenge to the investment in individualistic and volitional conception of autonomy: the self-sufficient liberal individual as a political subject lof will and consciousness) is constituted through the establishment of differentiating boundaries that stabilize its positioning, which in turn sustains the idea of freedom and supports the claim for rights. This identity-centered political map related directly to the conception of autonomy as a matter of control over our bodies. In this way, the boundaries that configure the autonomous individual establish the ontological status of the body as an enclosed, clear, and distinct object or entity or even, as in neoliberal frames, as an enterprise". 
2010[2006]:38). ¿Cómo se podría pensar en una agencia que no se desentienda de la dependencia fundamental que la funda? $\mathrm{Si}$ nuestros cuerpos dependen de los otros para sobrevivir y para florecer, ¿no habría una posibilidad de enfocarse en la interdependencia social, en lugar de en las libertades individuales, para pensar una política radical y plural, es decir, una política que no se limite a comprometerse con la libertad sino también con la igualdad y la justicia? ¿Es posible pensar la subjetividad y la política más allá del imaginario hegemónico neoliberal, abriendo así potenciales caminos para la resistencia desde un punto de vista democrático radical y plural?

\section{Por un feminismo radical y plural}

\section{a. Democracia radical y plural}

En Hegemonía y estrategia socialista, Ernesto Laclau y Chantal Mouffe proponen, a través de un análisis crítico del marxismo clásico y de la deconstrucción de ciertas categorías centrales de esta corriente de pensamiento, una alternativa para la izquierda a la que llaman "democracia radical y plural". Aunque estos autores no se centren exclusivamente en la teoría y la política feministas, sino que su reflexión abarca otras y diversas luchas contemporáneas propias de los "nuevos movimientos sociales", considero que la lógica política que proponen y su crítica al liberalismo pueden ser de gran ayuda para pensar el feminismo que estoy intentando proponer.

El punto de partida es la crisis de dos cuestiones clave para el marxismo clásico: del privilegio ontológico de la clase obrera y del ideal de una revolución que traerá una sociedad de la que habrán desaparecido los antagonismos. ${ }^{16}$ Esta crisis propia de

\footnotetext{
${ }^{16} \mathrm{Me}$ interesa resaltar aquí que la corriente liberal llega, con un diagnóstico diferente y de signo político contrario, a conclusiones similares respecto de la posibilidad de una voluntad colectiva homogénea en una sociedad no marcada por el conflicto. Con una visión "post-política", sostienen que la etapa de desarrollo económico y político que hemos alcanzado constituye un progreso en
} 
finales del siglo XX da lugar a una reflexión sobre el carácter plural y abierto de las luchas contemporáneas y la conflictividad como una dimensión inerradicable de la política. Considero aquí, junto a Mouffe, que el antagonismo es "una posibilidad siempre presente" (2009:13; la traducción es mía) $)^{17}$, es decir que la conflictividad es lo propio de lo político y que no puede ser erradicada. Esto se debe, siguiendo a nuestros autores, a la existencia de una forma de negatividad que no puede ser superada, en la medida en que la sociedad nunca constituye una totalidad suturada. El pluralismo propio de la contemporaneidad, lejos de ser superado, necesita ser mantenido a través del conflicto y el antagonismo que lo caracterizan. El conflicto, el antagonismo y la confrontación son la prueba de que la democracia está viva y habitada por el pluralismo. Dada la inerradicabilidad de las fuerzas antagónicas, cualquier fijación de un orden social será el efecto de una articulación temporal, parcial y precaria, cualquier forma de objetividad será una articulación hegemónica contingente o, mejor dicho, la expresión de una lucha hegemónica, la cristalización de relaciones de poder.

Dado que el campo de las identidades colectivas implica indefectiblemente la creación de un "nosotros" que se demarca de un "ellos", el desafío de la democracia liberal consiste en, justamente, establecer dicha relación de una forma que mantenga la emergencia del antagonismo a raya. El punto es cómo conjugar la inerradicabilidad del antagonismo con la posibilidad de un pluralismo democrático, de forma tal que la relación nosotros/ellos sea compatible con una democracia pluralista. Una cuestión fundamental a destacar aquí es que las luchas políticas no se fundan en identidades sustanciales, sino que, en la concepción de Laclau y Mouffe, el punto de partida radica en las demandas que se articulan equivalencialmente. El concepto de articulación viene

la evolución de la humanidad en el cual el "mundo libre" ha triunfado sobre el comunismo y que gracias al consenso obtenido a través del diálogo racional podrán implementarse los derechos humanos universales (Cfr. Mouffe, 2005:1).

17 "an ever-present possibility". 
a introducir aquí la idea de que esta práctica modifica la identidad de los elementos que entran en dicha relación; es decir que no se trata de elementos positivos definidos en sí mismos que entran en relación con otros elementos positivos definidos en sí mismos, sino que éstos se definen negativamente a través de una relación diferencial. Cuando entran en la cadena de equivalencias a través de la articulación, estos elementos (diferencias no articuladas) se transforman en momentos (posiciones diferenciales articuladas). Así, la práctica articulatoria es "toda práctica que establece una relación tal entre elementos, que la identidad de éstos resulta modificada como resultado de esa práctica" (Laclau y Mouffe, 2010:142-143). A través de la producción de una cadena de equivalencias que subvierte el carácter diferencial de los términos se produce una configuración que no se funda en un principio subyacente exterior a sí mismo, sino que es contingente, precaria y transitoria, pues la transición de momentos a elementos nunca se realiza completamente $y$, en ese sentido, ni las relaciones logran absorber las identidades, ni las identidades pueden constituirse plenamente. Siempre quedan elementos que no son articulados en la cadena discursiva (significantes flotantes) que hacen que proliferen los significados, desbordando todo punto nodal, que es lo que limita la cadena significante permitiendo que se produzca allí un sentido.

La articulación equivalencial puede tener lugar sólo una vez que se ha abandonado la concepción de la sociedad como totalidad y se ha asumido la apertura de lo social como constitutiva, pues si la sociedad fuera una totalidad plena y positiva, no habría necesidad de articulación equivalencial. Esto se inaugura, según Laclau y Mouffe, a partir del momento que, retomando la expresión de Tocqueville, los autores llaman "revolución democrática": un momento en el que se da una mutación en el imaginario político, un quiebre que permite el pasaje de una sociedad jerárquica y desigualitaria, regida por una lógica teológica política donde la voluntad divina funda el orden social, a una sociedad en la cual, a partir de la disponibilidad del discurso democrático y sus principios de libertad e igualdad, 
diversas formas de desigualdad pueden ser concebidas como formas de opresión ilegítima y, por tanto, como el sitio del antagonismo. El proyecto de una democracia radical y plural consiste en una etapa de profundización de la "revolución democrática" $y$, por lo tanto, se propone extender las luchas democráticas por la igualdad y la libertad a un número crecientemente amplio de relaciones sociales. Por lo tanto, la alternativa de izquierda debe establecerse al interior del campo de la "revolución democrática": su objetivo no es romper con la democracia liberal sino profundizar el momento democrático a través de la creación de equivalencias entre las distintas luchas.

La tarea de la izquierda no puede por tanto consistir en renegar de la ideología liberal democrática sino al contrario, en profundizarla y expandirla en la dirección de una democracia radicalizada y plural. [...] No es en el abandono del terreno democrático sino, al contrario, en la extensión del campo de las luchas democráticas al conjunto de la sociedad civil y del Estado, donde reside la posibilidad de una estrategia hegemónica de la izquierda (Laclau y Mouffe, 2010:222).

La democracia radicalizada se centra en la pluralidad y la apertura y pretende multiplicar los espacios políticos para impedir la concentración en un solo punto. En este sentido, la perspectiva tradicional de la izquierda que pretendía determinar a priori el sujeto de la revolución y los puntos de ruptura privilegiados encuentra sus límites en la formulación de la política hegemónica. De alguna forma, lo que se quiebra es la idea de que se puede plantear la resistencia en términos teleológicos, definiendo quiénes serán los sujetos que llevarán a cabo qué acciones con qué consecuencias. La superficie de emergencia de los antagonismos está constantemente subvertida por los efectos sobredeterminantes de otras, lo cual implica un constante desplazamiento de las lógicas sociales de unas esferas hacia otras. Así, cuando una lucha produce efectos de equivalencia con otras luchas, empieza a tomar 
forma el proyecto de una democracia radical y plural. Para que esto suceda, es menester que las cadenas de equivalencias se expandan y abarquen otras nuevas luchas, sin que esto haga que se pierda la autonomización de los espacios en los que cada lucha se constituye.

Para que esta lógica democrática se convierta en un proyecto hegemónico, es menester que, aparte del desplazamiento equivalencial a relaciones sociales cada vez más amplias, se pueda fundar algún punto nodal en torno al cual se pueda reconstituir el tejido social. El proyecto hegemónico requiere la gestión de la positividad de lo social y la integración de la articulación de las diversas demandas. El proyecto de una democracia radical y plural implica una dimensión socialista, en la medida en que plantea la abolición de las relaciones capitalistas de producción; sin embargo, no cree que esta sea la opresión constitutiva que, una vez abolida, abolirá todas las otras desigualdades. En este sentido, la autonomía de las distintas luchas, la multiplicación de los antagonismos y la construcción de los espacios donde estos emergen son condiciones necesarias para alcanzar aquel ideal clásico del socialismo que precisa, al mismo tiempo, ser ampliado y reformulado.

\section{Un feminismo radical y plural}

En su último libro, Butler analiza las "asambleas públicas", manifestaciones colectivas concertadas que cuestionan un cierto orden político. Se trata de asambleas, huelgas, vigilias y distintas formas de ocupación del espacio público como acción concertada que se encarna para demandar un conjunto de vidas más vivibles.

[C] uando los cuerpos se congregan en la calle, en la plaza o en otro espacio público (incluyendo los virtuales) están ejerciendo un derecho plural y performativo a aparecer, uno que afirma y establece el cuerpo en medio del campo político y que, en su función expresiva y significativa, emite una demanda corporal por un conjunto de condiciones económicas, sociales y políticas más vivibles, ya no afligidas 
por formas inducidas de precaridad (Butler, 2015:11, la traducción es mía). ${ }^{18}$

En estas manifestaciones, una pluralidad de cuerpos produce una asamblea pública y encarna un objetivo común sin que esto implique que esa pluralidad de cuerpos constituya un sujeto político unificado. Así, la aparición pública en conjunto no significa necesariamente que todos los cuerpos allí presentes estén de acuerdo con todo lo que se dice en nombre de dicha movilización. Cuando los cuerpos se encuentran en el espacio público para hacer presente su existencia plural y efectuar una serie de reclamos, están haciendo siempre algo más que eso: están demandando reconocimiento y ejerciendo el derecho a aparecer. En este sentido, toda protesta es, implícitamente, una demanda por el derecho de reunión, más allá de la cuestión puntual sobre la que verse dicha movilización. Es una demanda de cuerpos que piden ser oídos, que persisten en su existencia y cuestionan la distribución diferencial de la precaridad a través de una performatividad específica del cuerpo que ejerce un derecho que no es un derecho: el derecho a tener derechos. ${ }^{19}$ Ahora bien, ¿cómo se entiende esta acción performativa plural en la cual cuerpos "sin derechos" piden derechos? Aquí aparece un dilema: no podemos actuar sin apoyo $y$, sin embargo, muchas veces luchamos justamente por aquellos apoyos que nos permitirán actuar. La paradoja de la acción performativa plural en condiciones de precaridad es, justamente, actuar en nombre del apoyo sin apoyo.

18 "WJhen bodies assemble on the street, in the square, or in other public space (including virtual ones) they are exercising a plural and performative right to appear, one that asserts and instates the body in the midst of the political field, and which, in its expressive and signifying function, delivers a bodily demand for a more livable set of economic, social, and political conditions no longer afflicted by induced forms of precarity".

${ }^{19}$ Butler retoma el concepto del "derecho a tener derechos" de Hannah Arendt. Cfr. Arendt (1998). 
Para eso, es preciso correrse el eje de la moral neoliberal individualista que sostiene que cada sujeto es responsable de producirse a sí mismo y empezar a comprender que la situación de precarización no es individual sino compartida y que está inducida por fallas y desigualdades de las instituciones socioeconómicas y políticas. Si la moral neoliberal hace de cada uno el responsable de sí mismo y no de los otros y toma esa responsabilidad como la responsabilidad de volverse económicamente autosuficiente en condiciones donde la autosuficiencia está estructuralmente socavada, entonces es menester desmontar dicha forma de responsabilidad en favor de un ethos que afirme la dependencia, que comprenda la vulnerabilidad como un modo de relacionalidad y que, por lo tanto, pueda imaginar formas colectivas de abordar la precaridad, comprendida como fenómeno político. Esto implica una comprensión de la vulnerabilidad como una forma de activismo, como aquello que es movilizado por formas de resistencia que luchan por órdenes sociales y políticos más igualitarios.

¿Qué posibilidades se abren para la teoría y la política feministas a través la crítica a la política de la identidad y a la concepción (neo)liberal de la política y la subjetividad? ¿Existe para el feminismo alguna posibilidad que no lo subsuma a las políticas neoliberales y le devuelva, por así decirlo, su espíritu crítico, incluso contra hegemónico? ¿Y qué tipo de feminismo será ese? Si pensamos en una nueva política feminista que empiece con un entendimiento de la interdependencia, es decir, que pueda dar cuenta de la relación entre precaridad y performatividad, se abre un nuevo tipo de política que logra correrse del eje fundacional de la identidad. Si la precaridad es la condición contra la cual varios de los así llamados nuevos movimientos sociales luchan, eso no significa que pretendan superar la interdependencia. Por el contrario, la tarea es producir condiciones en las cuales la vulnerabilidad y la interdependencia sean vivibles. Así, se trata de producir nuevas formas de vida, formas de vidas vivibles. Como sostiene Butler, "cuando funciona, hay una instauración performativa de democracia radical" (Butler 
2015:218, la traducción es mía). ${ }^{20}$ La resistencia ocasiona una nueva forma de vida que se opone a la distribución diferencial de la precaridad en el nombre de vidas vivibles. Se trata de buscar, a través de la acción concertada que caracteriza la resistencia hoy en día, la producción de los principios de igualdad y de interdependencia, de forma tal que emerja una democracia más radical y más plural.

En los últimos años en Argentina, conceptos tales como sororidad, autonomía, empoderamiento, femicidio, ni una menos, nos mueve el deseo, y algunos que causan algo más de controversia en la sociedad como aborto libre, seguro y gratuito, visibilidad lesbiana, furia travesti, derecho migrante, entre otros, se oyen cada vez más, quiero decir con más frecuencia y con más fuerza. Proliferan nuevas agrupaciones de mujeres, lesbianas, travestis y trans, los espacios políticos tradicionales abren su sección de género, cada vez hay en las redes sociales más grupos destinados a compartir información y debatir problemáticas feministas, el movimiento sale a las calles, moviliza el espacio público y hasta lleva a cabo un paro de mujeres, aparecen lesbianas y trans/travestis en la televisión, las marchas del orgullo LGBTIQ+ son cada vez más multitudinarias, aumentan las denuncias por violencias y abusos, emerge un nuevo periodismo feminista, y cantidad de otros acontecimientos que dan cuenta de que el feminismo ha ganado terreno en el imaginario social, cultural, político y económico.

Ahora bien, es cierto que, tal como sostiene Fraser, dentro de este feminismo tan amplio emergió, a lo largo de las últimas décadas, un feminismo neoliberal que sirvió para dar aires progresistas a políticas de precarización. Sin embargo, la reducción de todo el feminismo a este feminismo empresarial nos impide ver otros feminismos que también han emergido en el último tiempo y que resisten a las tecnologías neoliberales de gobierno, aunque muchas veces se vean atrapados por ellas. Estos son los

20 " when it works, there is a performative enactment of radical democracy". 
movimientos feministas que aquí me interesan y que quiero analizar.

El 3 de junio de 2015 se llevó adelante la primera movilización por "Ni una menos" ${ }^{21}$, una consigna que sintetiza las formas de decir basta a la violencia machista y a su máximo exponente, el femicidio. Con una segunda marcha el 3 de junio de 2016 y una tercera el 3 de junio de 2017, la fecha quedó inscripta de manera definitiva en el calendario del feminismo en Argentina. El 19 de octubre de 2016 se convocó a un paro de mujeres que, a la espontaneidad con que el movimiento había salido a las calles en las otras ocasiones, le sumó la capacidad de articular una gran diversidad de experiencias sindicales, sociales y políticas en una sucesión de asambleas en las cuales se definieron colectivamente métodos de decisión y de trabajo y que produjeron un manifiesto común en el cual se plasmaron las denuncias, las demandas y los deseos. El 8 de marzo de 2017 se produjo un segundo paro de mujeres, esta vez en el marco de un paro internacional, que también contó con una serie de asambleas previas convocadas por el colectivo Ni Una Menos.

Hay aquí una cuestión fundamental: pensar las movilizaciones feministas en términos de un paro es una forma de darse a la tarea de discutir dicha medida como herramienta no exclusiva de los sindicatos, ni siquiera del trabajo reconocido como tal, lo cual implica pensar qué formas de trabajo son las que pueden verse involucradas en un paro de tareas. Esto opera un corrimiento respecto de la clase trabajadora como sujeto privilegiado de la resistencia (como quedó ya explicitado a través de la crítica posmarxista de Laclau y Mouffe) y abre el juego político al feminismo que, de alguna forma u otra, se inscribe en una tradición de izquierda. Por otro lado, esas movilizaciones en las cual las mujeres (o, estrictamente hablando, los sujetos feminizados) salen del ámbito doméstico o privado para

\footnotetext{
21 "Ni una menos, ni una muerta más" son los versos de Susana Chávez, una poetisa y activista mexicana asesinada en 2011 por denunciar los crímenes contra las mujeres en México.
} 
manifestarse en el espacio público expresan, si seguimos la lectura butleriana, aparte del listado de demandas concretadas plasmadas en el documento unificado y producido colectivamente, una demanda por el derecho a tener derechos, una forma de aparición y de demanda por el reconocimiento. Una tercera cuestión a resaltar es el trabajo de articulación que se ha producido entre demandas de distinto orden: algunas estructurales vinculadas al desempleo o a los empleos precarizados, endeudamiento, derechos de las migrantes, derecho a la tierra y la vivienda, educación, disidencia sexual, brecha salarial, activismo gordo, economía popular, violencia contra las mujeres, transexualidad, entre otros, fueron los temas y problemas que se debatieron para dar lugar, finalmente, a un documento único que contemplara todas las demandas y a una movilización única en la que participaran todas las organizaciones feministas así como personas no agrupadas.

La marea feminista que se gestó en los últimos años en Argentina permite entrever un feminismo acerca del que quiero arriesgar la hipótesis de llamarlo radical y plural, en los dos sentidos que explicitaré a continuación. ${ }^{22}$ En primer lugar, se trata de un feminismo que no parte de la identidad ni del requisito de un sujeto único y determinado para constituirse. En ese sentido, es

\footnotetext{
${ }^{22}$ Aquí se impone la aclaración de que cuando hablo de un feminismo radical y plural no me refiero al feminismo radical que surgió en los años sesenta y setenta en Estados Unidos. Esa corriente feminista se denominó a sí misma radical porque pretendía buscar la raíz de las relaciones de dominación entre varones y mujeres. Así, el lema "lo personal es político" da cuenta de una de las ideas centrales para este feminismo, para el cual hasta lo más íntimo estaba atravesado por relaciones de poder. Si bien esta corriente del feminismo es clave para comprender el patriarcado como estructura de opresión que el poder masculino despliega sobre las mujeres en amplios y variados contextos de la vida, su oscilación entre la concepción cultural de la dominación masculina y el determinismo biológico ha propiciado algunas críticas. Entre ellas, el problema del patriarcado universal que se sigue de la desestimación de las condiciones históricas y sociales, lo cual legitimaría su validez y perpetuaría la opresión de las mujeres, por un lado, y la discusión en torno a la transexualidad y el transgénero, por el otro. Al respecto, ver: Jaggar (1983) y Stryker (2008).
} 
un feminismo que recoge la crítica al feminismo mainstream (blanco, heterosexual, de clase media y occidental) para poder abarcar distintos tipos de problemáticas asociadas a distintas subjetividades y comunidades. El vector que moviliza es la constatación de una situación de precaridad inducida diferencialmente que es compartida y el propósito del movimiento es producir vidas más vivibles. Movilizando la vulnerabilidad sin pretender por ello superarla bajo la figura de "sujetas" empoderadas y, por tanto, autónomas (en los sentidos liberales de estos términos), este feminismo se propone crear redes comunitarias de contención y agencia política. Así, campañas como "Estamos para nosotras" o el trabajo llevado a cabo por las Socorristas son la prueba de que las políticas paternalistas no constituyen una solución definitiva. Esto no significa que no se necesiten políticas públicas ni leyes estatales; si bien "no todos nuestros sueños caben en sus urnas", los derechos son necesarios para promover una nueva distribución, acaso menos diferencial, de la precaridad. Y aquí aparece el segundo sentido en que este feminismo puede pensarse como radical y plural: se trata de un feminismo que, articulando equivalencialmente una pluralidad de demandas inicialmente aisladas, produce performativamente un grupo unificado (en términos de una movilización única y de la confección de un documento de demandas comunes), con la tarea de radicalizar los principios democráticos de libertad e igualdad. Una vocación de ocupación callejera llevó a la ocupación del espacio público, con movilizaciones que aliaron a los cuerpos en las calles, ejerciendo así no sólo las demandas específicas plasmadas en el documento único, sino también el derecho a aparecer y constituyendo una marea multitudinaria cuyo potencial, creo, todavía no puede cifrarse. En este sentido, considero que una tarea a futuro de este feminismo radical y plural será dar lugar a la imaginación de nuevas formas políticas, si no quiere quedar reducido a la lengua jurídica liberal de los derechos. 


\section{Conclusiones}

Empecé el presente artículo señalando un debate en el que, a mi entender, lo que estaba en juego era la posibilidad de un feminismo anti-(neo)liberal, con el propósito de responder afirmativamente a dicha posibilidad y de embarcarme en la tarea de analizar qué tipo de feminismo anti-(neo)liberal podía trazarse hoy en día, en tiempos de democracias neoliberales y gobernanza a través de la precarización. Así como la modalidad neoliberal produjo una suerte de alianza con un feminismo empresarial, para dar aires progresistas a políticas de austeridad, del mismo modo creo que pueden verse intersticios a través de los cuales ciertas técnicas de gobierno pueden volverse contra ellas mismas. Y este es, quizás, el caso de la precarización como gubernamentalidad, si pensamos, como aquí lo hago, que al tomar la precaridad como punto de partida de la reflexión política, podemos llegar a nuevos puertos.

Mi hipótesis, siguiendo a Judith Butler y a Leticia Sabsay, es que esta nueva forma de pensar la agencia política (a partir de la precaridad) permite varios desplazamientos respecto del imaginario (neo)liberal que dan lugar a lo que aquí llamé un feminismo radical y plural, retomando la expresión de Ernesto Laclau y Chantal Mouffe. Para esto, partí de la crítica a la noción moderna liberal de la subjetividad y a los problemas que suscita pensar la resistencia en términos de las políticas de la identidad y luego me centré en la idea de una democracia radical y plural y en las movilizaciones recientes en el marco de la democracia neoliberal, lo cual me dio el marco propicio para desarrollar un feminismo de articulaciones en Argentina en los últimos años.

Tal como fue anunciado por Laclau y Mouffe, nada garantiza que una articulación vaya a ser de izquierda o de derecha y probablemente el feminismo en Argentina hoy se enfrente a ese problema. La masividad del feminismo tiene un matiz sumamente positivo, en la medida en que permite cuestionar lugares antes considerados como verdades absolutas, pero corre asimismo el riesgo de vaciarse de sentido y no ser más 
que un maquillaje aliado del capitalismo financiero más salvaje. Es por eso que considero necesario que el feminismo se aboque a una tarea de radicalización y pluralización del movimiento que permita trazar nuevas articulaciones con otros movimientos sociales y políticos para constituir una verdadera alternativa de izquierda hegemónica. Esto no significa aceptar la idea de Nancy Fraser de que ciertas demandas del feminismo son "meramente culturales", sino por el contrario comprender el carácter material de la distribución diferencial de la precaridad.

\section{Referencias bibliográficas}

ARENDT, Hannah. Los orígenes del totalitarismo. Madrid: Taurus, 1998.

ButLER, Judith. Merely Cultural. Social Text, 52-53, 1997, pp.265-277.

. Precarious Life. The Powers of Mourning and Violence. Londres/Nueva York, Verso, 2006.

. Gender Trouble. Feminism and the Subversion of Identity. Londres/Nueva York, Routledge, 2007.

. Deshacer el género. Madrid, Paidós, 2010.

. Frames of War. When is Life Grievable? Londres/Nueva York, Verso, 2010.

. Notes toward a performative theory of assembly. Cambridge, Massachusetts/Londres: Harvard University Press, 2015.

. "Rethinking Vulnerability and Resistance", en: Vulnerability in resistance. Druham/Londres, Duke University Press, 2016.

; Gambetti, Zeynep; Sabsay, Leticia. "Introduction", en: Vulnerability in resistance. Druham/Londres, Duke University Press, 2016.

De Beauvolr, Simone. El segundo sexo. Buenos Aires, Debolsillo, 2012.

FRASER, Nancy. Iustitia Interrupta. Reflexiones críticas desde la posición "postsocialista". Bogotá, Siglo del Hombre Editores, 1997. 
, Nancy . Fortunas del feminismo. Del capitalismo gestionado por el Estado a la crisis neoliberal. Madrid, Traficantes de sueños, 2015.

, Nancy. "El final del neoliberalismo 'progresista". en Sin permiso. República y socialismo, también para el siglo XXI. http://www.sinpermiso.info/textos/el-final-del-neoliberalismoprogresista, 2017.

JAGGAR, Alison. Feminist Politics and Human Nature. New Jersey, Rowman \& Allanheld, 1983.

LACLAU, Ernesto y Mouffe, Chantal. Hegemonía y estrategia socialista. Hacia una radicalización de la democracia. Buenos Aires, Fondo de Cultura Económica, 2010.

LOREY, Isabell. Estado de inseguridad. Gobernar la precariedad. Madrid, Traficantes de sueños, 2016.

LOWENKRON. Consent and Vulnerability: some intersections between child sexual abuse and the trafficking in persons for sexual exploitation. cadernos pagu (45), Campinas, SP, Núcleo de Estudos de Gênero-Pagu/Unicamp, diciembre 2015.

MoufFE, Chantal. The return of the political. Londres/Nueva York, Verso, 2005.

, Chantal. On the political. Londres/Nueva York, Routledge, 2005.

. The Democratic Paradox. Londres/Nueva York, Verso, 2009.

. Agonistics. Thinking the world politically. Londres/Nueva York, Verso, 2013.

Preciado, Paul B. Testo Yonqui. Sexo, drogas y biopolítica. Buenos Aires, Paidós, 2014.

; Saez, Javier. "Prólogo" a Lenguaje, poder e identidad. Madrid, Síntesis, 2004.

SABSAY, Leticia. Permeable Bodies: Vulnerability, Affective Powers, Hegemony. Vulnerability in resistance. Druham/Londres, Duke University Press, 2016. 
. The political imaginary of sexual freedom. Subjectivity and power in the new sexual democratic turn. Londres, Palgrave Macmillan, $2016 b$.

Soley-Beltran, Patricia; Preciado, Paul B. Abrir posibilidades. Una conversación con Judith Butler. Lectora, 13, 2006, pp.217-239.

STRYKER, Susan. Transgender History. Boston, Seal Press, 2008.

$\mathrm{RICH}$, Adrienne. Heterosexualidad obligatoria y existencia lesbiana. DUODA. Revista d'Estudis Feministes n $10,1996$.

SCHILD, Verónica. Feminismo y neoliberalismo en América Latina. New Left Review 96, enero-febrero, 2016, pp.63-79.

WitTIG, Monique. The straight mind and other essays. Boston, Beacon Press, 1992. 